Review

\title{
Current Status and Progress of Specific Laboratory Examination Methods of Active Tuberculosis Infection Diagnosis
}

\author{
Xuyang Chen ${ }^{1}$, Kan Wang ${ }^{2,3}$, Hongying $\mathrm{Li}^{1}$, Lixin Hong ${ }^{1}$, Jinghua $\mathrm{He}^{1}$ \\ ${ }^{1}$ Outpatient Department, Zhujiang Hospital, Southern Medical University, Guangzhou, Guangdong 510280, China. \\ ${ }^{2}$ Department of Instrument Science and Engineering, School of Electronic Information and Electrical Engineering, Shanghai Jiao \\ Tong University, Shanghai 200240, China. \\ ${ }^{3}$ Shanghai Engineering Research Center for Intelligent diagnosis and treatment instrument, Shanghai 200240, China. \\ Corresponding author. E-mail: hjh5258@foxmail.com; wk_xa@163.com
}

Received: Nov. 8, 2017; Accepted: Mar. 28, 2018; Published: Mar. 30, 2018.

Citation: Xuyang Chen, Kan Wang, Hongying Li, Lixin Hong, and Jinghua He, Current Status and Progress of Specific Laboratory Examination Methods of Active Tuberculosis Infection Diagnosis. Nano Biomed. Eng., 2018, 10(I): 79-86.

DOI: 10.5101/nbe.v10i1.p79-86.

\begin{abstract}
Active tuberculosis infection is a major health concern in the world. Each year, millions of people die of tuberculosis, especially in third-world countries. Though the World Health Organization has recently reported the rate of mortality by this disease is declining by $3 \%$ yearly, active tuberculosis infection is still endangering human health seriously. In addition, there are many people who have a latent tuberculosis infection, and these people do not seek treatment because they have no clinical symptoms. It is true that current specific laboratory examination approaches are capable of diagnosing active tuberculosis infection promptly and accurately. But sensitivity and specificity of current diagnostic approaches are at a low level. However, the development of new nanomaterials allows more scientists to combine diagnostic methods with nanotechnology. Recently, a novel Nanodisk mass spectrometry method has been reported. Mycobacterium tuberculosis-specific peptides are enriched using an antibody-conjugated nanodisk, allowing for rapid, quantitative detection of the serum-specific antigen for active tuberculosis infection. This method overcomes the shortcomings of poor sensitivity and long turnaround time associated with current diagnostic approaches. This review discusses the current status and progress of specific laboratory examination methods of active tuberculosis diagnosis and compares the newest diagnostic techniques.
\end{abstract}

Keywords: Active tuberculosis (TB); Diagnosis; Nanodisk mass spectrometry (Nanodisk-MS)

\section{Background}

Tuberculosis (TB) is a major world health concern. It spreads quickly, has high incidence, and is difficult to diagnose. Though major medical advances have been achieved in the last century, TB is still a significant cause of morbidity and mortality [1]. According to the statistics, there are still more than
8 million people suffer from the disease worldwide. In the last century, the World Health Organization (WHO) declared TB is a global emergency [2]. TB is a highly persistent infection caused by Mycobacterium tuberculosis (Mtb). Mtb invades human tissues and causes tissue damage. Mtb can be present as an active tuberculosis infection as well as a latent TB infection (LTBI). Once infected with $M t b$, people 
have the highest risk of developing TB disease within the first two years. Despite the advance of medical therapies, Mtb causes over 1.5 million deaths in the world each year [3, 4]. In addition, approximately one third of the global population has a LTBI $[4,5]$. Most people, with latent TB which is associated with $M t b$ infection, have no symptoms, but they are at high risk of developing active tuberculosis in their lifetime [5]. Patients with active TB infection are an important source of infection, because of the presence of $M t b$ in their sputum. Mtb can spread through the droplets. If healthy person come into contact with these droplets containing $M t b$, they may be infected with $M t b$. Mtb is likely to be suppressed, if one infected with $M t b$ has a strong immunity. Finally, Mtb may be present as a LTBI. The WHO strongly recommends systematic testing and anti-TB treatment to reduce the number of active TB cases. The WHO has proposed a strategic target of ending TB by the year 2030 [6]. If we use reliable diagnostic testing methods to identify active tuberculosis infection in time, the target will be achieved on schedule. It is a fact that current specific laboratory examination methods can diagnose active tuberculosis infection timely and accurately. But the sensitivity and specificity are poor. Due to the factors, clinicians may need to repeat the test several times. So the weak points of current diagnostic methods need to be improved.

A new generation of methods for diagnosing active TB infection is needed. The fields of nanomaterials and nanotechnology have developed quickly during the past 20 years, with good magnetic orientation and biocompatibility, such as gold nanoparticles, silica nanoparticles, and iron oxide nanoparticles [44, 45]. Due to the unique physical and chemical properties of nanomaterials, this area of research has been popular. Recently, more and more scientists are committed to diagnosing and treating diseases with nanomaterials and nanotechnology. For example, nano-drug delivery systems are being developed to diagnose and treat cancer with good results. Recently, a novel method that uses nanoparticles has been developed to diagnose active TB infection [36]. Experimental data have shown that the sensitivity, specificity, and accuracy of active TB infection diagnosis with nanoparticles are satisfactory. Maybe this method overcomes some of the existing dilemmas. Additionally, imaging techniques combined with nanoparticles, such as gold nanoparticle-based magnetic resonance imaging, can improve the accuracy of active TB infection diagnosis.
The use of nanomaterials may dramatically improve the sensitivity and specificity of active TB diagnosis in the future.

\section{Currently Used Laboratory Examination Approaches}

Effective treatment of TB relies on accurate diagnosis. Timely and accurate identification of individuals and communities with active TB infections is very important for controlling and managing TB. The gold standard of diagnosing active TB is the identification of the organism from a cultured clinical sample. The tuberculosis skin test (TST) and two different gamma interferon assays are classic diagnostic tests. Though both are widely used in the world, there are some limitations that cannot be ignored. These tests can only provide indirect information about active TB infection [7-13]. The aim of this review is to introduce the current status and recent progress of specific laboratory examination of active TB diagnosis. The TST, Gamma interferon assays and Nanodisk mass spectrometry (Nanodisk-MS) method are shown in Fig. 1.

\section{Tuberculosis skin test}

The TST has been used worldwide for over 100 years [14]. It is the first assay that be selected to screen individual who may have active tuberculosis infection. TST is based on the response of the human immune system to special-TB proteins [16, 17]. TST uses purified protein derivative and observes the size of local induration at the inoculation site after two to three days of TB protein exposure [7, 15]. Then, according to the size of induration to determine whether individual is infected with Mtb. So TST is very convenient and suitable for large-scale population early screening. In addition, it has other advantages, such as cost-effectiveness and simplicity; however, it also has several known limitations. With the developments of modern medicine, we have more ways to prevent Mtb infection. However, current diagnostic techniques often deliver false-positive or false-negative results [15]. A false positive result can occur with the TST because of cross-reaction with nontuberculosis mycobateria. In addition, most children had the Bacillus Calmette Guerin (BCG) vaccination when they were born, which may cause a false positive result in the TST [79]. The BCG vaccination can stimulate production of anti-TB antibodies (anti-TB Ab). Therefore, the antiTB Ab will react with TB-antigen of the TST, even 


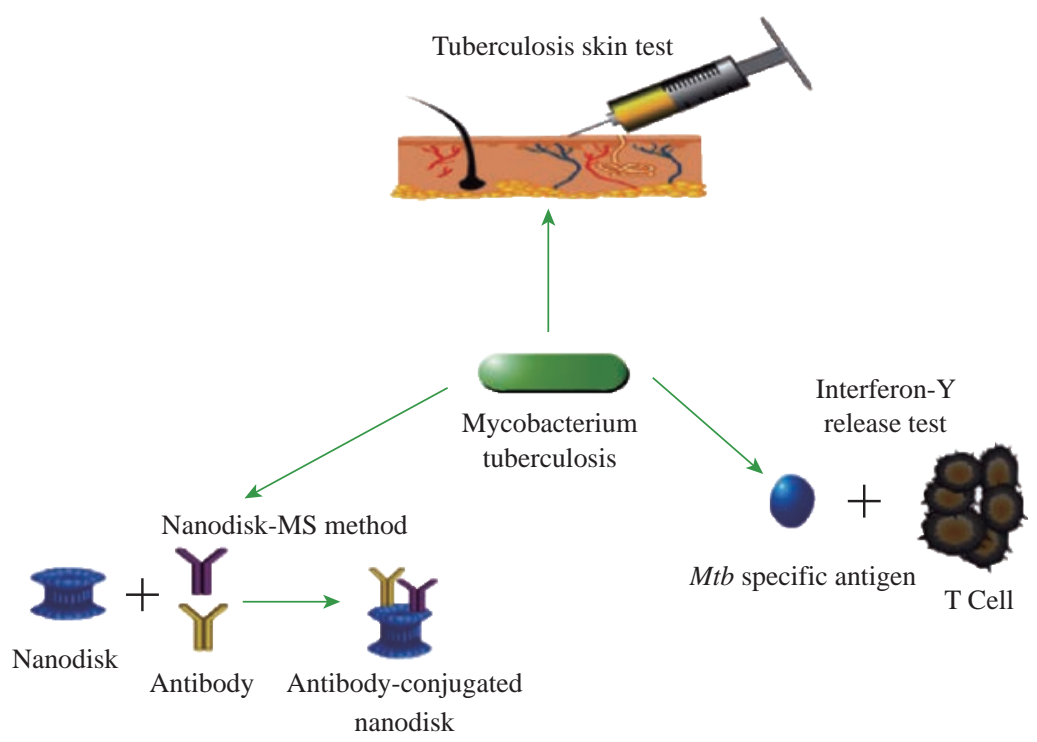

Fig. 1 The classic and newest specific laboratory examination methods of active TB infection: TST, Interferon-y release assay and Nanodisk-MS method.

if the patient does not have an active Mtb infection. Thus, it may cause a false-positive result. Additionally, individuals undergoing immunosuppressive treatments will have false-negative results when they undergo TST [10]. Since the body's immune system has been suppressed with immunosuppressive treatments, the body cannot produce anti-TB Ab to bind with TBantigen. Further, inappropriate storage of tuberculosis antigen can cause false TST negative results. Over time, the shortcomings of the TST are becoming more obvious, and these factors limit its use.

\section{Interferon-y release assay}

There are two other assays that detect the specific immune response to Mycobacterium tuberculosis (Mtb) in vitro. They are also used widely in the world yet [1820]. One is the Quanti FERON-TB Gold In-tube assay, and the other is the T.SPOT-TB assay. The bioplausible principle of both assays is that the production of INF- $\gamma$ will increase after peripheral blood cells are exposed to the specific M. tuberculosis antigen [21, 22]. In the Quanti FERON-TB Gold In-Tube assay, T cells are exposed to early secreted antigenic target 6 (ESAT6) and culture filtrate protein 10 (CFP-10), which are specific to Mtb. After exposure, T cells are incubated overnight. The test does not require isolation of a single T cells from whole blood. During the incubation, the production of INF- $\gamma$ is monitored to measure the T-cell response to the Mtb-specific antigen. Finally, scientists confirm active infection with the number of INF- $\gamma$ produced by $T$ cells. In the T.SPOT-TB assay, the serum sample is exposed to ESAT-6 and CFP-10.
In this case, the peripheral blood mononuclear cells must be isolated from the whole blood before testing, because the number of INF- $\gamma$-producing cells on coated plates are monitored [23]. This is different from the Quanti FERON-TB Gold In-Tube assay. Currently, the accepted cut-off point is five-cell population. However, more studies should be conducted to evaluate if the cut-off is appropriate for clinical samples.

Both assays have been in use for over 10 years [24]. Compared with the TST, the interferon- $\gamma$ release assay has several advantages, which have been previously reported [18, 25-27]. Most importantly, the interferon- $\gamma$ release assay does not cross-react with BCG, which may occur with TST. Therefore, the interferon- $\gamma$ release assay can be used in individuals who have received the BCG vaccine. In addition, the use of specific proteins improves the specificity and sensitivity of detection. Moreover, the use of the Interferon- $\gamma$ release assay is more cost-effective than TST as a screening test for high-risk populations [28-31].

Not surprisingly, some disadvantages of the assays do exist, such as an increase in the complexity of TB diagnosis. Historically, this has limited the use of the Interferon- $\gamma$ release assay. If we use the Interferon- $\gamma$ release assay to detect $M t b$ infection, peripheral blood samples must first be obtained, and the remaining detection steps must be peformed in the laboratory. However, it may be difficult to obtain blood samples from children, the elderly and unhealthy adults. Currently, it is difficult for clinical staff to judge whether a patient is infected with $M t b$ when 
using Interferon- $\gamma$ release assay. The sensitivity and specificity of these assays should be further evaluated with more clinical samples. The T.SPOT-TB assay requires the isolation of PBMCs before testing. Specifically, blood cell separation technology directly affects the test results. Interferon- $\gamma$ release assays are based on $\mathrm{T}$ cell-mediated immunity. However, neither assay can accurately distinguish active tuberculosis infection from latent tuberculosis infection [26, 28, 32, 33]. The largest limitation of Interferon- $\gamma$ release assays is that the average cost of both assays is $\$ 20$ higher than TST [15].

\section{Other laboratory examination methods}

There are several methods that should also be evaluated as tools to diagnosis active tuberculosis infection. Though culture of Mtb improves sensitivity, it has a long turnaround time [28]. Sputum smear microscopy has poor sensitivity [28], so sputum smear often need to be check repeatedly many times. This may delay the diagnosis of active TB and it is not useful to diagnose extrapulmonary tuberculosis (EPTB). Additionally, it can be difficult to obtain sputum samples in children. However, once Mycobacterium tuberculosis is found in sputum smear, TB infection can be confirmed. The adenosine deaminase assay (ADA) is mainly used in the diagnosis of tuberculosis pleurisy. It is a relatively sensitive and specific test for tuberculosis pleurisy. The ratio of ADA in the effusion to ADA in serum is more meaningful than the ADA value itself [34, 35]. Some molecular level detection methods, such as line-probe assays, PCR-based method, also are used to diagnose active TB. In addition, Gene Xpert MTB/RIF system is used to not only diagnose active $\mathrm{TB}$, but also provide information on drug resistance. In summary, currently used methods have limitations that are difficult to overcome. Therefore, there is an urgent need for a rapid and accurate diagnostic method for detecting active TB infection.

Of course, non-specific laboratory examination indexes including Blood Cell Sedimentation Rate, C-reactive protein also are used to diagnose active tuberculosis infection. But a large number of factors can make the non-specific laboratory indexes change, so they only can act as auxiliary diagnosis characters. In addition, a few of active TB infection patients have no obvious symptoms, so it is difficult for doctors to distinguish between active tuberculosis infection and inflammation and tumor. If the doctor just relies on laboratory examination methods, they may not make an accurate and timely diagnosis. Hence, clinicians will take a piece of patient's tissue specimens for pathological examination. So the pathological examination also plays an important role on active TB diagnosis.

\section{New Approach for Diagnosing Active TB: Nanodisk-MS Method}

The traditional diagnostic approaches mentioned above have many limitations. However, with the rapid development of nanomaterials, new opportunities for increasing the diagnostic accuracy for active TB infection are possible. Due to the unique physical and chemical properties of nanomaterials, this area of research has attracted many scientists in recent years. Recently, a rapid, blood-based, quantitative method, with high sensitivity and high specificity, has been reported to improve the accuracy of detecting active TB infection. The biggest innovation of the method is to combine an antibody-conjugated Nanodisk with high throughput MALDI time-of-flight mass spectrometry to improve sensitivity and specific detection of active TB infections [36]. The fundamental chemistry of the method is shown in Fig. 2. The experimental data shows that the assay has satisfactory sensitivity and specificity- This novel method can be used to diagnose patients with pulmonary tuberculosis (PTB) or extrapulmonary tuberculosis (EPTB), even for immunosuppressed individuals [36]. If it is true that the new method is superior to current approaches, the accuracy, sensitivity, and specificity of active TB diagnosis may be dramatically improved in the future.

The new method consists of three parts. The first part is the use of two $M t b$-specific protein peptides: 10-KDa culture filtrate protein (CFP-10) and 6-KDa early secretory antigenic target (ESAT6) [36]. Free serum concentrations of both target peptides in healthy humans are low. However, both peptides can be produced by active mycobacterial strains. Once infected, these proteins are secreted into circulating blood in the early stages of infection. Over time, both peptide free serum concentrations will increase. Therefore, CFP-10 and EAST-6 free serum concentrations can be used to diagnose active TB infection. Thus, the rapid quantitative detection of $M t b$ specific proteins is very important. Both peptides are highly specific to $M t b$ [42], and the concentration of both peptides in serum is related to active TB infection 


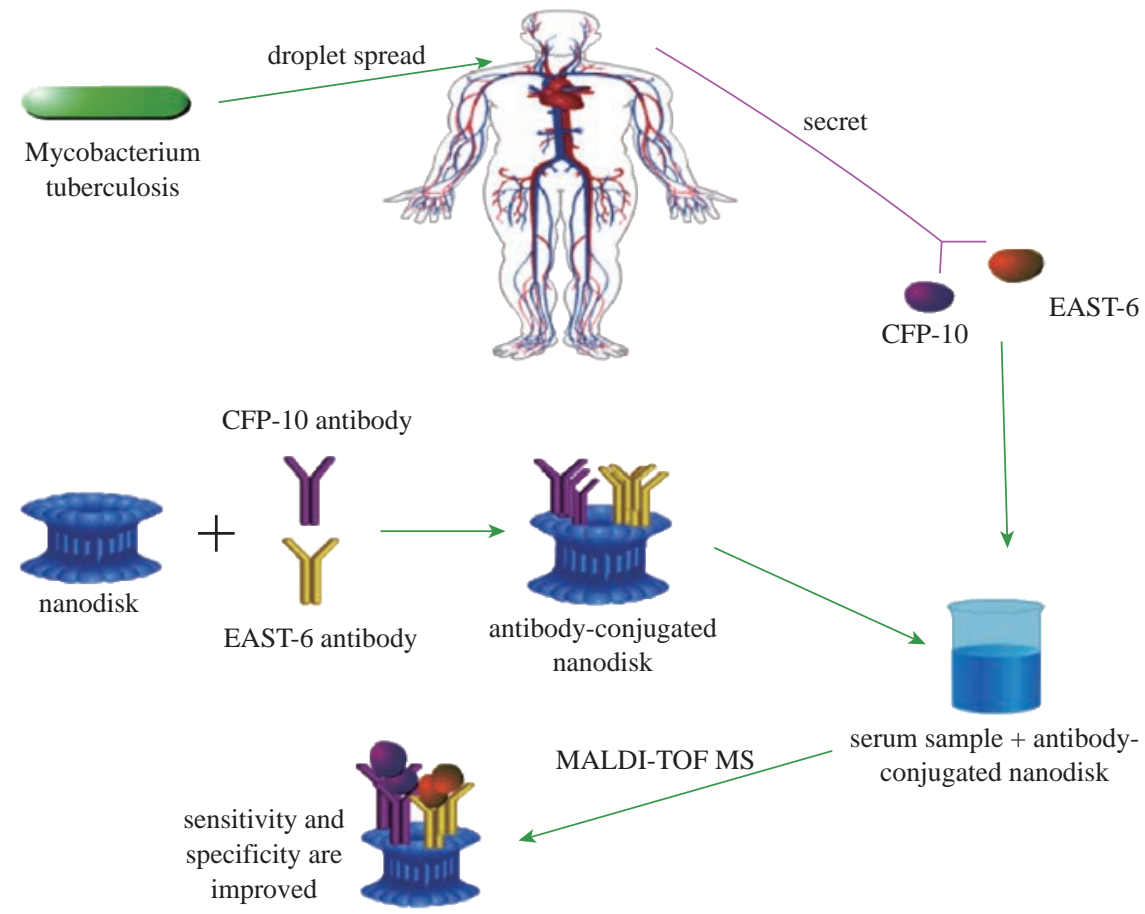

Fig. 2 Schematic of the diagnostic steps of the Nanodisk-MS method.

[36, 37]. Therefore, the determination of the free serum concentrations of these proteins can be used in the early diagnosis of $M t b$. Additionally, they can be used to diagnose all $M t b$ infections, including PTB and EPTB [46]. However, some nontuberculosis mycobacterium (NTM) can produce homologs of EAST-6 and CFP10 , which may interfere with the diagnosis of TB in clinical settings [47]. EAST-6 has little homology, but CFP-10 has some homology with proteins secreted by some NTM strains [37]. Fortunately, these strains are a relatively rare form of $\mathrm{TB}$; therefore, the potential for interference is minimal. Thus, the free concentrations of both protein peptides in serum can be used as an early signal of active TB infection [42]. To resolve the problem, tryptic peptides are selected to disrupt protein complexes in serum, releasing bound peptides. Some studies have shown that tryptic peptides can distinguish EAST-6 and CFP-10 from other homologs produced by other nontuberculosis mycobacteria (NTM) [38, 39]. In addition, the use of microwave-irradiation can facilitate the digestion of bound peptides in serum within 20 min [40]. Finally, MALDI-TOF MS analysis of bound peptides can enhance detection accuracy.

The second part of the method is the use of nanodisks. As stated previously, the concentrations of free peptides are very low, especially in the early stages of infection. Thus, an antibody-conjugated nanodisk is used to enrich both target peptides to increase the concentrations of free peptides. The free serum concentrations of target peptides are dramatically improved by increasing peptide desorption/ionization with nanoparticles. This may be due to UV absorbance properties and the thermal confinement effect of nanoparticles. After enrichment, the free peptides are detected by high-throughput MALDI time-of-flight mass spectrometry (MALDI-TOF MS), and the MS signal of CFP-10 and EAST-6 peptides is enhanced by nanodisk enrichment [36].

The enrichment process occurs in two steps. The first step is to place the antibody-conjugated nanodisk into a serum sample. After enrichment, several of antigen-antibody complexes will form. The second step is the enhancement of the MS signal with the nanodisk. Thus, after microwave digestion and nanodisk enrichment, the Nandisk-MS method can detect target peptides at lower concentrations. The research shows that Nanodisk-MS method has high sensitivity both in PTB (87.5\%) and EPTB (85.7\%) [36]. However, this method also requires the collection of blood samples prior to testing, but sometimes it is difficult for clinicians to collect the patient's blood samples, especially if the patient is a child, an elderly person, an obese person, etc.

The nanomaterial selected for the nanodisk platform was silica nanoparticles, because silica nanoparticles can increase the MS signal stability and be readily 
Table 1 Comparsion of active TB diagnostic methods

\begin{tabular}{cccc}
\hline Approach & Advantage & Disadvantage \\
\hline Tuberculosis skin test & Cost-effective, simple,convenient & High false positive rate \\
Interferon-y Release Assay & No cross-reaction with BCG; No follow-up visit $\quad$ The sensitivity and specificity are unreliable & Low sensitivity; long analysis time \\
Nanodisk-MS method & High sensitivity and specificity & Expensive; Has not been widely used \\
Other nanoparticle-based methods & High sensitivity and specificity & Has not been transformed into clinical use
\end{tabular}

modified as needed.

Today, MALDI-TOF MS is a routine detection technology in many hospitals and labs. The third part of the Nanodisk-MS method is the use of a ubiquitous clinical instrument. The use of MALDI-TOF MS may promote a rapid clinical transformation for the Nanodisk-MS assay [41].

Additionally, this method may be used to monitor patient responses to anti-TB drug therapies, allowing for appropriate and timely adjustment of the treatment plan. On further exploration of the Nanodisk-MS method, scientists may discover new approaches to diagnose other diseases, such as lung cancer.

Though the Nanodisk-MS method shows great potential, it is still in the experimental validation phase and has not been implemented in clinical settings. Even the sensitivity and specificity of the new method are better than current approaches, it is too early to predict its performance in comparison with current methods. So we do not need to overestimates the usefulness of the new method. The reliability of the Nanodisk-MS method needs further study [42].

\section{Other Nanoparticle-Based Detec- tion Methods for Mtb Infection Diagnosis}

In recent years, a few of new methods based on nanoparticles have been developed to diagnose $M t b$ infection by detecting specific DNA of $M t b$. In addition, some nanoparticle-based detection methods for immunoassay of $M t b$ also have been developed by many research groups. Until now, there are about 35 nanoparticle-based detection methods which almost cover all clinical aspects of tuberculosis [48]. Some experimental data have shown that the sensitivity and specificity of these methods are satisfactory. These methods have the potential to overcome the shortcoming of current approaches.

Though these methods have some advantage and high potential, no one has been transformed into clinical use successfully [48]. None of the developed approaches has target tuberculosis in children. Many of methods for detecting specific DNA of $M t b$ require the amplification of target DNA before detection. So it needs more time and cost. Additionally, the synthesis and functionalization steps of nanoparticle are complex, so it is not suitable for large-scale production at this stage (Table 1).

\section{Conclusions}

In summary, the current diagnostic approaches for active TB infection provide information regarding the host immune response to $M t b$-specific antigen. However, the information is indirect and often inaccurate. Moreover, the sensitivity and specificity of current diagnostic approaches are poor. In an effort to improve TB diagnosis, a Nanodisk-MS method has been developed, and it allows for rapid, quantitative detection of serum specific antigen for active TB infection. This method may improve the status of low sensitivity of current diagnostic approaches. The advantage of this method is its compatibility with high-throughput MALDI -MS technology, which is used routinely in many labs and hospitals. The factor may be able to promote the clinical transformation of new method. In addition, there are around 35 detection approaches based on nanoparticle for $M t b$ infection diagnosis. We should make an effort to promote the clinical transformation of these methods in the future. The steps of nanoparticle synthesis and functionalization should streamlined for large-scale 
production.

Active TB is a major global health burden, due to the lack of an effective vaccine and the emergence of the drug-resistant strains, especially in third-world countries. It cause over millions of deaths worldwide each year. If the nanoparticle-based detection methods can be transformed into clinical use, it may help to change this situation.

\section{Acknowledgments}

We are grateful for the financial support by the National Natural Scientific Foundation of China (Grant No. 81571835, 81671737, 61503246 and 81672247), Shanghai Science and Technology Fund No. 15DZ2252000, Shanghai Municipal Commission of Economy and Informatization Technology Fund NO. XC-ZXSJ-02-2016-05.

\section{Conflict of Interests}

The authors declare that no competing interest exists.

\section{References}

[1] A. Gonzalez-Garcia, J. Fortun, E. Elorza Navas, et al., The changing epidemiology of tuberculosis in a Spanish tertiary hospital (1995-2013). Medicine (Baltimore), 2017, 96: e7219.

[2] R. Shegokar, L. Al Shaal, and K. Mitri, Present status of nanoparticle research for treatment of tuberculosis. $J$ Pharm Pharm Sci, 2011, 14: 100-116.

[3] C. Seshadri, N. Sedaghat, M. Campo, et al., Transcriptional networks are associated with resistance to Mycobacterium tuberculosis infection. PLoS One, 2017, 12: e0175844.

[4] R.M. Houben, P.J. Dodd, The global burden of latent tuberculosis infection: A re-estimation using mathematical modelling. PLOS Medicine, 2016, 13: e1002152.

[5] H. Getahun, A. Matteelli, R.E. Chaisson, et al., Latent Mycobacterium tuberculosis infection. $N$ Engl J Med, 2015, 372: 2127-2135.

[6] M. Uplekar, D. Weil, K Lonnroth, et al., WHO's new end TB strategy. Lancet, 2015, 385: 1799-1801.

[7] J. H. Joncas, R. Robitaille, and T. Gauthier, Interpretation of the PPD skin test in BCG-vaccinated children. Can Med Assoc J, 1975, 113: 127-128.

[8] M. Farhat, C. Greenaway, M. Pai, et al., False-positive tuberculin skin tests: What is the absolute effect of BCG and non-tuberculous mycobacteria? Int J Tuberc Lung Dis, 2006, 10: 1192-1204.

[9] R.E. Watkins, R. Brennan, and A.J. Plant, Tuberculin reactivity and the risk of tuberculosis: A review. Int $J$ Tuberc Lung Dis, 2000, 4: 895-903.

[10] F. Deck, J. Guld, The WHO tuberculin test. Bull Int Union Tuberc, 1964, 34: 53-70.

[11] American Thoracic Society, Targeted tuberculin testing and treatment of latent tuberculosis infection. MMWR Recomm Rep, 2000, 49: 1-51.

[12] M. Pai, C.M, Denkinger, S.V. Kik, et al., Gamma interferon release assays for detection of Mycobacterium tuberculosis infection. Clin Microbiol Rev, 2014, 27: 3-20.

[13] V. Herrera, S. Perry, J. Parsonnet, et al., Clinical application and limitations of interferon-gamma release assays for the diagnosis of latent tuberculosis infection. Clin Infect Dis, 2011, 52: 1031-1037.

[14] P.Q. Edwards, L.B. Edwads, Story of the tuberculin test from an epidemiologic viewpoint. Am Rev Respir Dis, 1960, 81(1) Pt 2: 1-47.

[15] R.T. Horvat, Gamma interferon assays used in the diagnosis of tuberculosis. Clin Vaccine Immunol, 2015, 22: 845-849.

[16] O. Manuel, D. Kumar, QuantiFERON-TB gold assay for the diagnosis of latent tuberculosis infection. Expert Rev Mol Diagn, 2008, 8: 247-256.

[17] A. Lalvani, Diagnosing tuberculosis infection in the 21st century: new tools to tackle an old enemy. Chest, 2007, 131: 1898-1906.

[18] G.H. Mazurek, J Jereb, A. Vernon, et al., Updated guidelines for using Interferon Gamma Release Assays to detect Mycobacterium tuberculosis infection-United States, 2010. MMWR Recomm Rep, 2010, 59: 1-25.

[19] C.M Denkinger, K. Dheda, and M. Pai, Guidelines on interferon-gamma release assays for tuberculosis infection: concordance, discordance or confusion? Clin Microbiol Infect, 2011, 17: 806-814.

[20] H.R. Mujakperuo, R.D. Thompson, and D.R. Thickett, Interferon gamma release assays and the NICE 2011 guidelines on the diagnosis of latent tuberculosis. Clinical Medical, 2013, 13: 362-366.

[21] M.J. Fenton, M.W. Vermeulen, S. Kim, et al., Induction of gamma interferon production in human alveolar macrophages by Mycobacterium tuberculosis. Infect Immun, 1997, 65: 5149-5156.

[22] A.M. Gallegos, J.W. van Heijst, M. Samstein, et al., A gamma interferon independent mechanism of CD4 T cell mediated control of $\mathrm{M}$. tuberculosis infection in vivo. PLoS Pathog, 2011, 7: e1002052.

[23] P. Andersen, M.E. Munk, J.M. Pollock, et al., Specific immune-based diagnosis of tuberculosis. Lancet, 2000, 356: 1099-1104.

[24] G.H. Mazurek, J. Jereb, P. Lobue, et al., Guidelines for using the QuantiFERON-TB Gold test for detecting Mycobacterium tuberculosis infection, United States. MMWR Recomm Rep., 2005, 54: 49-55.

[25] M. Bocchino, B. Bellofiore, A. Matarese, et al., IFNgamma release assays in tuberculosis management in selected high-risk populations. Expert Rev Mol Diagn, 2009, 9: 165-177.

[26] G. Ferrara, M. Losi, R.D. Amico, et al., Use in routine clinical practice of two commercial blood tests for diagnosis of infection with Mycobacterium tuberculosis: A prospective study. Lancet, 2006, 367: 1328-1334.

[27] M.G. Madariaga, Z. Jalali, and S. Swindells, Clinical utility of interferon gamma assay in the diagnosis of tuberculosis. J Am Board Fam Med, 2007, 20: 540-547.

[28] Y. Dai, Y. Feng, R. Xu, et al., Evaluation of interferongamma release assays for the diagnosis of tuberculosis: an updated meta-analysis. Eur J Clin Microbiol Infect Dis, 2012, 31: 3127-3137.

[29] G. Redelman-Sidi, K.A. Sepkowitz, IFN-gamma release assays in the diagnosis of latent tuberculosis infection among immunocompromised adults. Am J Respir Crit Care Med, 2013, 188: 422-431.

[30] M. Sester, G. Sotgiu, C. Lange, et al., Interferon-gamma release assays for the diagnosis of active tuberculosis: a systematic review and meta-analysis. Eur Respir J, 2011, 
37: 100-111.

[31] A. Kowada, Cost-effectiveness of interferon-gamma release assay for entry tuberculosis screening in prisons. Epidemiol Infect, 2013, 141: 2224-2234.

[32] M. Pareek, M. Bond, J. Shorey, et al., Communitybased evaluation of immigrant tuberculosis screening using interferon gamma release assays and tuberculin skin testing: Observational study and economic analysis. Thorax, 2013, 68: 230-239.

[33] A.N. Aggarwal, R. Agarwal, D. Gupta, et al., Interferon gamma release assays for diagnosis of pleural tuberculosis: a systematic review and meta-analysis. $J$ Clin Microbiol, 2015, 53: 2451-2459.

[34] Q.L. Liang, H.Z. Shi, K. Wang, et al., Diagnostic accuracy of adenosine deaminase in tuberculous pleurisy: A metaanalysis. Respir Med, 2008, 102: 744-754.

[35] J. Klimiuk, R. Krenke, Role of biomarkers in making the diagnosis of tuberculous pleurisy. Pneumonol Alergol Pol, 2011, 79: 288-297.

[36] C. Liu, Z. Zhao, J. Fan, et al., Quantification of circulating Mycobacterium tuberculosis antigen peptides allows rapid diagnosis of active disease and treatment monitoring. Proc Natl Acad Sci U S A, 2017, 114: 3969-3974.

[37] C.C. Lai, C.K. Tan, C.H. Chou, et al., Increasing incidence of nontuberculous mycobacteria. Taiwan, 20002008. Emerg Infect Dis., 2010, 16: 294-296.

[38] V.A. Simossis, J. Heringa, The PRALINE online server: optimising progressive multiple alignment on the web. Comput Biol Chem, 2003, 27: 511-519.

[39] J. van Ingen, R. de Zwaan, R. Dekhuijzen, et al., Region of difference 1 in nontuberculous Mycobacterium species adds a phylogenetic and taxonomical character. $J$ Bacteriol, 2009, 191: 5865-5867.

[40] W. Sun, S. Gao, L. Wang, et al., Microwave-assisted protein preparation and enzymatic digestion in proteomics. Mol Cell Proteomics, 2006, 5: 769-776.

[41] R. Patel, MALDI-TOF mass spectrometry: Transformative proteomics for clinical microbiology. Clin Chem, 2013,
59: 340-342.

[42] C.M. Denkinger, J. Grenier, J. Minion, et al., Promise versus reality: optimism bias in package inserts for tuberculosis diagnostics. J Clin Microbiol, 2012, 50: 2455-2461.

[43] A. Muller, H. Bogge, F.L. Sousa, et al., Nanometer-sized molybdenum-iron oxide capsule-surface modifications: External and internal. Small, 2007, 3: 986-992.

[44] G. Kim, R. O'regan, and S. Nie, Biomedical nanotechnology for molecular imaging, profiling and drug targeting. Proceedings of the 27th Annual International Conference of the Engineering in Medicine and Biology Society. Shanghai, China, Jan. 17-18, 2005: 714-716.

[45] C. Lang, D. Schulaer, and D. Faivre, Synthesis of magnetic nanoparticles for bio- and nanotechnology: genetic engineering and biomimetics of bacterial magnetosames. Macromol Biosci, 2007, 7:144-151.

[46] T.T. Feng, C.M. Shou, L. Shen, et al., Novel monoclonal antibodies to ESAT-6 and CFP-10 antigens for ELISAbased diagnosis of pleural tuberculosis. Int J Tuberc Lung Dis, 2011, 15: 804-810.

[47] S.M. Arend, K.E. van Meijgaarden, K. de Boer, et al., Tuberculin skin testing and in vitro $\mathrm{T}$ cell responses to ESAT- 6 and culture filtrate protein 10 after infection with Mycobacterium marinum or M. kansasii. J Infect Dis, 2002, 186: 1797-1807.

[48] E.S. Hesham, A. Asma, A.T. Mohamed, et al., Nanodiagnostics for tuberculosis detection. Expert Review of Molecular Diagnostics, 2017, 5: 427-443.

Copyright $₫$ Xuyang Chen, Kan Wang, Hongying Li, Lixin Hong, and Jinghua He. This is an open-access article distributed under the terms of the Creative Commons Attribution License, which permits unrestricted use, distribution, and reproduction in any medium, provided the original author and source are credited. 